\title{
Crystallographic texture evolution in high-density polyethylene during uniaxial tension
}

\author{
D. Li ${ }^{\text {a }}$, H. Garmestani ${ }^{\text {a,* }}$, S.R. Kalidindi ${ }^{\text {b }}$, R. Alamo ${ }^{\mathrm{c}}$ \\ ${ }^{\mathrm{a}}$ Department of Mechanical Engineering, Center for Materials Research \& Tech, FAMU-FSU College of Engineering, Room 229, \\ 2525 Pottsdamer Road, Tallahassee, FL 32310-6046, USA \\ ${ }^{\mathrm{b}}$ Department of Materials Engineering, Drexel University, Philadelphia, PA 19104, USA \\ ${ }^{\mathrm{c}}$ Department of Chemical Engineering, FAMU-FSU College of Engineering, Tallahassee, FL 32310, USA
}

Received 14 August 2000; received in revised form 27 October 2000; accepted 27 October 2000

\begin{abstract}
This paper presents experimental measurements of crystallographic texture evolution in high-density polyethylene subjected to very large strains in uniaxial tension (up to a true strain of 2.1). The measurements presented here differ from prior studies in three important aspects: (1) The initial texture in the sample is quite strong with a large fraction of the crystallites oriented in an unstable orientation with the crystal $c$ axis perpendicular to the tensile axis of the sample. (2) Rigorous methods of texture analyses, based on spherical harmonics, have been applied to produce "complete, recalculated" pole figures based on diffraction data from five incomplete pole figures. (3) The measurements were performed while the samples were kept in the deformed state. The results presented here provide several new insights into texture development in tensile straining of high-density polyethylene to large strains. There are at least three distinct preferred orientations: (i) a component with (001) aligned along the extension axis, (ii) a component with (011) aligned close to the extension axis, and (iii) a component with (010) aligned along the extension axis. Note that only the first component has been reported to be stable at high strains in previous studies. The rate of texture evolution in the present study is significantly lower than that reported in previous studies. It was also observed that the natural relaxation of strain following the tensile loading had a significant impact on the texture in the sample. It was observed that the relaxation process mitigated or eliminated the second and third preferred texture components described above, while strengthening the first. (C) 2001 Elsevier Science Ltd. All rights reserved.
\end{abstract}

Keywords: High-density polyethylene; Uniaxial extension; Texture analysis

\section{Introduction}

Polyethylene (PE) is a widely used semi-crystalline polymer with a range of applications in packaging, house wear, sporting goods, and biomedical uses, among several others. By varying the catalyst and/or the polymerization process, polyethylenes can be obtained having markedly different chain structures, solid-state structures and physical mechanical properties [1]. The many useful applications and relatively low cost of ethylene led, since their initial synthesis in the 1930s, to continuous research and development in these materials. Interest always arises to understand the relation between molecular, structural and processing variables and their end-use properties [2]. These fundamental studies have grown exponentially in the last 10 years since the advent of polyolefins made with the new generation of metallocenetype catalysts [3].

\footnotetext{
* Corresponding author. Tel.: +1-850-644-5993; fax: +1-850-644-9281.

E-mail address: garm@magnet.fsu.edu (H. Garmestani).
}

Based on molecular structure, the polyethylenes are divided into four different types. The linear or high-density polyethylene (HDPE) contains no short chain branching (SCB) or a very small fraction of SCB. Copolymerization of ethylene with a 1-alkene type comonomer yields polyethylenes with a larger fraction of SCB, the so-called linear low density polyethylenes (LLDPE). High-pressure homopolymerization (LDPE) or copolymerization of ethylene in tubular reactors leads to polyethylenes and ethylene copolymers with both types of branching, SCB and long chain branching (LCB). The introduction of branching or non-crystallizing units into the chain leads to a very rapid and continuing decrease of crystallinity with increasing side group content. Crystallinity and morphological features of the polyethylenes are probably the most important factors affecting their properties [4]. Apart from the interplay between molecular variables and the resulting crystalline structure after processing, understanding the basic deformation mechanisms has also been a subject of intense interest. The historical process of these studies was reviewed by Lin 
and Argon [4]. The deformation processes are expected to be a function of the initial morphology. Thus, although general deformation paths could be ascribed to semicrystalline polymers, the details of the deformation mechanisms are expected to change based on the initial crystallinity and crystalline structure developed on processing. It is, thus, not surprising that full understanding of the molecular mechanisms underlying the deformation of crystalline polymers has proven to be elusive. Theoretical treatments of the deformation process are always attractive as a means to predict the behavior of the material under load. However, many of these treatments have focussed on the deformation of a pure phase (crystal or amorphous) without taking into account the role of connectivity between these phases. For example, the Eyring-type activated processes to describe tensile properties of polyethylenes was restricted to the amorphous regions [5-8]. Nevertheless this model was qualitatively successful in describing the dependence of the draw ratio with molecular weight.

There is a strong interest in understanding the microstructural details of the crystalline regions and their evolution during the various thermo-mechanical treatments used in the processing of polyethylenes. There is also interest in understanding the deformation on the crystallographic scale. An important microstructural detail of the crystalline region is the distribution of the crystal lattice orientations in the given sample (also referred to as crystallographic texture or simply texture). The wide-angle X-ray diffraction pole figure analysis provides stereographic projection of diffraction planes as a function of their direction within the sample. In this work, we carried out a detailed pole figure analysis of the deformation process of the PE with the most simple molecular structure, the HDPE. The rapid crystallization conditions used are similar to those of many commercial processes.

The crystalline phase of $\mathrm{PE}$ is orthorhombic with lattice parameters $a=0.741 \mathrm{~nm}, b=0.495 \mathrm{~nm}$ and $c=0.255 \mathrm{~nm}$ [9]. The polymer chains lie parallel to the $c$-axis, [001] with strong covalent bonds along the chain and weaker van der Waals bonds between the chain segments. It should also be noted that the $c$-axis represents the closest packed direction in this crystal structure. The bonding nature in PE imparts a strong anisotropy to properties at the crystallite level. Consequently, texture plays an even more important role in controlling properties in high-density polyethylene.

Bartczak and co-workers [11,12] produced a highly textured (quasi single-crystal) sample of HDPE by employing extremely large strains in the channel-die and studied its mechanical behavior. Samples were cut from the textured material and subjected to tensile, compressive, and simple shear deformation in different directions, which were chosen to probe the deformation resistances of specific potential slip mechanisms. These authors reported that the easy slip mode in crystalline PE is chain slip on (100)[001], with chain slip on (010)[001] and (110)[001] being frequent alternatives. In deformation orientations requiring transverse slip, evidence was found for activation of (100)[010], (010)[100], and (110)[11̄0] transverse slip systems. Note that the combination of all of these slip systems provides only four independent slip systems; the crystal is incapable of length changes along the $c$-axis with the activation of these slip systems alone. Although deformation twinning and/or martensitic transformation can provide the fifth independent mode of plastic deformation in these crystals, there has not been any evidence of significant extent of these modes during PE deformation. It is generally assumed that shearing in the interlamellar amorphous regions provides the additional degree of freedom for plastic deformation in PE [13].

Deformation textures have been measured in HDPE in a number of deformation modes including uniaxial tension [10,13-18], uniaxial compression [11,19-22], biaxial extension [23] and simple shear [24]. Texture evolution in each of these cases exhibits a complex path suggesting that different mechanisms of deformation are prevalent in the material at different ranges of imposed strain in any particular mode of deformation. The specific deformation mechanisms responsible for these complex paths of texture evolution are, however, not yet clearly identified or understood. The assumption of cylindrical geometry and, consequently, the expansion of orientation distribution functions in a Legendre series is not adequate for the orthorhombic symmetry of polyethylene. Furthermore, in a number of studies, the measurements themselves are not "complete", because spherical harmonic techniques (or equivalent) have not been used in the analyses of pole figures from raw data obtained by X-ray diffraction.

The texture of polyethylene during uniaxial tension has been previously studied. Krause and Hosford [15] presented a detailed account of texture evolution in HDPE in uniaxial tension. They did not, however, report the initial texture of the sample. The measurements reported by these authors indicate that the PE crystals at low strains (about a true strain of 0.55 ) rotate such that the $c$-axis is about $35^{\circ}$ with the extension axis and the $a$-axis is normal to the extension axis. At higher strains, however, they report that the crystals rotate such that the $c$-axis becomes gradually parallel to the tensile axis with deformation. By about a true strain of 1.8 , they report that an extremely strong $c$-axis fiber texture develops accounting virtually for all of the crystals in the deformed PE. Krause and Hosford are, however, unclear about which slip/twinning mechanisms are responsible for this texture evolution. They surmise that the early texture development may be due to twinning and transverse slip on (100)[010] and suggest that the final texture path may be attributed to some of the chain slip mechanisms either by themselves or in combination with chain unfolding on (110) or (010) planes.

Pazur et al. [14] studied the texture evolution in extruded films. In this study, the film had an initial texture with the $a$ axis preferentially oriented in the plane containing the extension direction and transverse directions and the $b$-axis 


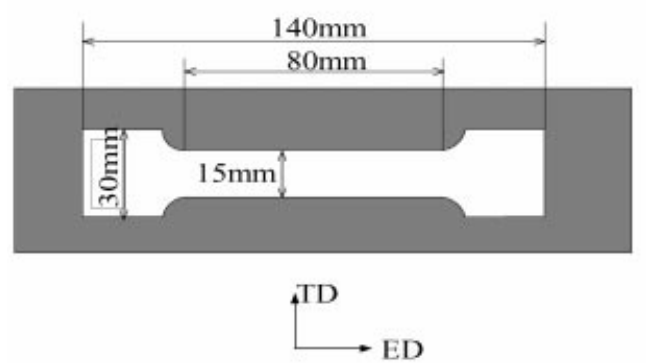

(a)

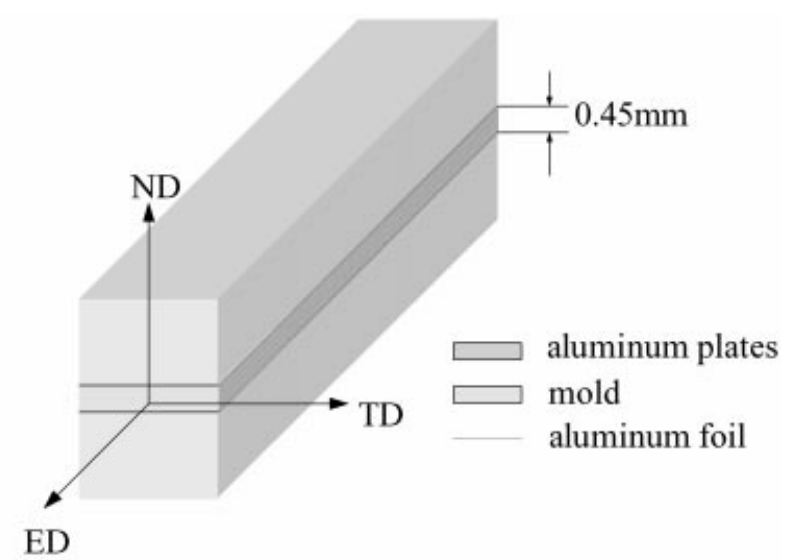

(b)

Fig. 1. (a) Schematic geometry of the polyethylene melt compression mold. (b) Device diagram for melt compression operation. ED, ND, TD are extension direction, normal direction and transverse direction, respectively.

strongly oriented along the film normal. It was reported that as the draw ratio was increased, the $c$-axis gradually realigned itself with the extension direction to introduce a fibrillar-type morphology. Butler et al. [16] investigated the cold drawing behavior of unoriented linear HDPE using simultaneous small and wide-angle X-ray scattering during deformation. No discernible lattice rotations occurred until the yield point was reached. Fine chain slip, most probably on the (100) $\{001\}$ slip system [20], was observed at the yield point.

It should be noted that in most of the studies mentioned, the texture measurements were conducted after allowing the sample to relax. Simultaneous SAXS/WAXS measurements were carried out by a few investigators [16,20,21,25,26] during in situ deformation. However, the pole figures were not constructed in any of these works. Relaxation is an inherent mechanism of deformation in polymers. As soon as the load is taken off the sample in the deformation experiments, relaxation processes initiate automatically. These can produce large unloading strains, especially in tensile loaded samples, and potentially cause significant changes in the underlying texture in the sample. Therefore, the textures reported in previous studies [10-15,19,22-24] correspond to "deformed and relaxed" textures in the samples, and may not represent accurately the "deformation only" texture in the sample.

There are also currently no theoretical or computational models that can provide good predictions of the preferred orientations in deformed PE. Because of the lack of five independent slip modes, the classical Taylor-type models [17] that have demonstrated good success in describing cubic metallic crystals cannot be extended easily to the $\mathrm{PE}$ case. It is necessary to formulate other averaging procedures to get reasonable solutions, let alone accurate solutions. Parks and Ahzi [27] provided the first solution with their modified Taylor formulation of crystal plasticity theory for fully crystalline polymers that takes into account the inextensibility of the PE crystal along the $c$-axis in determining the local stress and strain in the crystal. Although the prediction from this model yielded a strong $c$-axis fiber texture, it did not predict the two distinct regimes of texture evolution suggested by Krause and Hosford. Lee et al. [13] proposed a composite model that incorporated both the crystalline phase and the amorphous phase. This model has been reported to yield more realistic stress-strain curves. The model also does not predict the two distinct regimes of texture evolution described above. The predicted texture from this model, however, shows a much weaker $c$-axis component compared to the prediction by Parks and Ahzi [27]. In fact, the model of Lee et al. [13] predicts that the $c$ axis is misoriented from the tensile axis by a small angle, as evidenced by a small hole in the center of the predicted $c$ axis pole figure for this deformation path.

In an effort to resolve some of the issues described above, we have recently undertaken a detailed experimental study to document the evolution of crystallographic texture in rapidly crystallized HDPE in tension to large strains. We have applied rigorous methods of texture analyses based on generalized spherical harmonics that are now available to generate complete pole figures from a set of incomplete pole figures measured by X-ray diffraction [28]. Furthermore, we have attempted to document the deformation textures in HDPE under load, without being significantly affected by the relaxation processes.

\section{Experimental methods}

\subsection{Materials and sample preparation}

The material used in this study was a commercial linear HDPE made by the Phillips process. The weight-average 


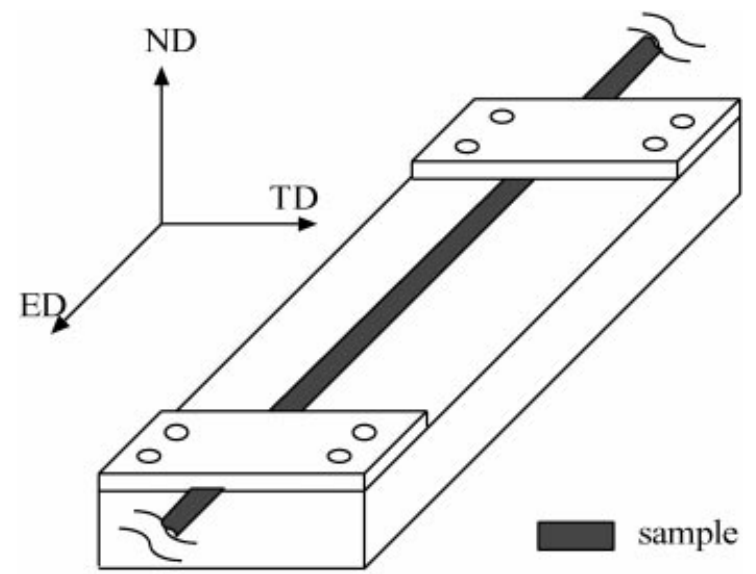

Fig. 2. Schematic diagram of the device used to maintain the specimen at a constant extension level.

molecular weight $\left(M_{\mathrm{w}}\right)$ of this polyethylene is $127,000 \mathrm{~g} /$ mol and the polydispersity, $M_{\mathrm{w}} / M_{\mathrm{n}}$, is 13.3 . The polyethylene pellets were placed in a mold that was positioned between two aluminum plates lined with aluminum foil. Fig. 1 shows a schematic illustration of the mold. Samples were prepared by compression molding in a Carver press at a temperature of about $175^{\circ} \mathrm{C}$ and a pressure of $150 \mathrm{~atm}$. The plate system was held in the molten state at $175^{\circ} \mathrm{C}$ for about $10 \mathrm{~min}$ and it was further submerged in tap water $\left(23^{\circ} \mathrm{C}\right)$. The dumbbell-shaped tensile specimens with a thickness of $0.45 \mathrm{~mm}$ were $140 \mathrm{~mm}$ long with gage dimension of $80 \mathrm{~mm} L \times 15 \mathrm{~mm} W$.

The melting temperature of the quenched specimens is $129^{\circ} \mathrm{C}$ as measured in a Perkin Elmer DSC-7 calorimeter at $10^{\circ} \mathrm{C} / \mathrm{min}$. From the observed heat of fusion, and using $290 \mathrm{~J} / \mathrm{g}$ as the heat of fusion per mole of pure crystal [29], the degree of crystallinity of the as prepared sample is $70 \%$.

\subsection{Mechanical tests}

The mechanical tests were carried out using a Model 1011 Instron tensile machine. The samples in this study were deformed at ambient temperature $\left(24-26^{\circ} \mathrm{C}\right)$, at an extension rate of $5 \mathrm{~mm} / \mathrm{min}$, to true strain levels of 0.11 , $0.7,1.2,1.8$, and 2.1 , respectively. Yielding was initiated at a strain of 0.11 , while failure occurred at about a strain of 2.1. The other strain levels $(0.7,1.2,1.8)$ were chosen evenly between the yield point and failure. For each strain level of interest, the actuator was stopped after reaching the desired strain level and while under load, a special fixture (Fig. 2) was attached to the sample to maintain it at the constant extension level. The sample with the attached fixture was then taken out of the Instron machine and placed directly in the X-ray goniometer to document the texture in the sample.

\subsection{X-ray diffraction}

Crystallographic texture in the undeformed and the deformed samples were measured by X-ray diffraction using a Philips X'Pert PW3040 MRD X-ray diffractometer, equipped with a pole figure goniometer, operating at $40 \mathrm{kV}$ and $45 \mathrm{~mA}$ and employing $\mathrm{Ni}$ filtered $\mathrm{Cu} \mathrm{K} \alpha$ radiation. Incomplete pole figures were obtained for the projections of sample orientation with respect to the incident beam, with $\psi$ (defined as the tilt angle) varying from 0 to $85^{\circ}$ in $5^{\circ}$ intervals and $\phi$ (defined as the rotation) varying from 0 to $360^{\circ}$ in $5^{\circ}$ steps. The following reflections were analyzed to determine texture components: (110), (200), (020), (011), (201) (diffraction angle $2 \theta=21.59,24.03,36.30,39.75$, 43.05 , respectively). The diffraction data were corrected for geometric defocusing and background X-ray intensity using standard procedures. A harmonic algorithm implemented in the software package, the preferred orientation package from Los Alamos (popLA) [30], was used to calculate the sample crystallite orientation distribution from which the experimental pole figures were reconstructed. The procedure is explained briefly below.

The total intensity of diffracted X-rays [31] at any point $(\alpha, \beta)$ on the $(h k l)$ pole figure is:

$P_{(h k l)}(\alpha, \beta)=\frac{1}{2 \pi} \int_{0}^{2 \pi} f(\Psi, \Theta, \phi) \mathrm{d} \Gamma$

Here the Euler angle set $\Theta=\alpha, \Psi=\beta, \Gamma$ denote the path through the orientation distribution (OD) corresponding to rotation about the $(h k l)$ pole. Expansion of Eq. (1) in a series of spherical harmonic functions yields

$P_{(h k l)}(\alpha, \beta)=\sum_{l=0}^{\infty} \sum_{m=-l}^{l} Q_{l m} P_{l}^{m}(\cos \alpha) \mathrm{e}^{\mathrm{i} m \beta}$

where $P_{l}^{m}(\cos \alpha) \mathrm{e}^{\mathrm{i} m \beta}$ is a spherical harmonic function; $l$ is often called the "order" of the spherical harmonic function. Further,

$Q_{l m}=\sum_{n=-l}^{l} W_{l m n} P_{l}^{n}(\cos \xi) \mathrm{e}^{-\mathrm{i} n \eta}$

where $W_{l m n}$ are the coefficients of the spherical harmonic functions, and $\xi$ and $\eta$ are the polar coordinates of the $(h k l)$ pole in the crystal coordinate system.

By measuring pole figures of multiple geometrically independent poles, we can obtain a set of linear simultaneous equations that can be solved for the $W_{l m n}$. For polyethylene, with an orthorhombic crystal structure, it is recommended to use at least five such pole figures. Once $W_{l m n}$ have been calculated, Eqs. (2) and (3) can be used to recalculate pole figures. We are not aware of any previous work that used these methods to study in situ deformation of polyethylenes.

\section{Results}

Fig. 3 shows the true stress-true strain curve computed from the measured load-displacement data obtained from 


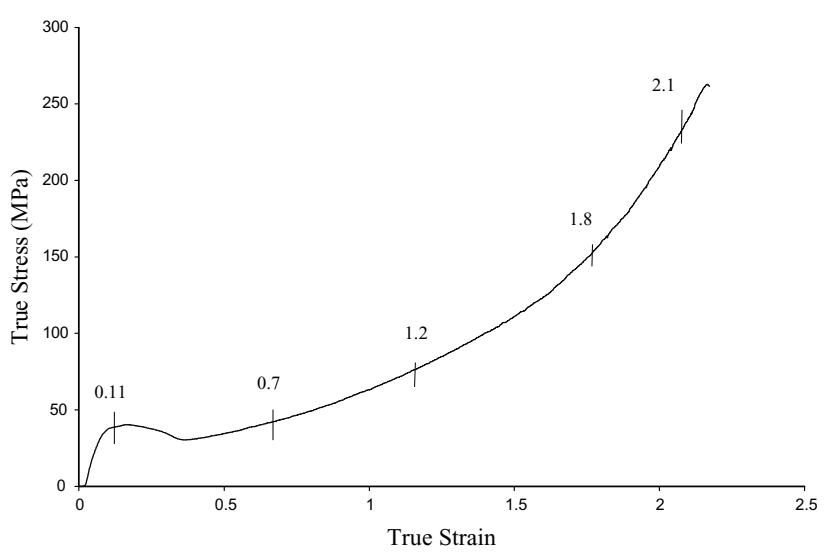

Fig. 3. Plot of true stress against true strain for rapidly quenched HDPE sample.

the tension test to large extensions. The curve indicates two distinct regimes. The initial elastic regime extends up to a strain of about 0.11 and is followed by a regime of plastic flow from the yield point to fracture.

Using a very small interval $\left(0.002^{\circ}\right)$ in the $2 \theta$ angle range, a fine scan plot of diffracted intensities was obtained from the HDPE sample. This scan is shown in Fig. 4. The diffraction patterns were indexed using the unit cell of orthorhombic polyethylene. This figure also shows some reflection corresponding to the strongest peak of the monoclinic phase, the (001) peak, at the position of $2 \theta=19.47^{\circ}$. However, the contribution from the monoclinic phase is very small, compared with other peaks from the orthorhombic crystals. Thus, the WAXS data were interpreted only on the basis of the main orthorhombic contributing phase, although some martensitic transformation may occur, as reported in other works [21].

The texture evolution in the samples during uniaxial extension is represented by (100), (010), and (001) pole figures in Fig. 5. The pole figures corresponding to strains of $0.0,0.11,0.7,1.2,1.8,2.1$ are presented in this figure. All figures were plotted in a stereographic projection with a $\log$ intensity scale. The orientation distribution function (ODF) analysis was performed using popLA [30], which produces normalized and recalculated pole figures for important texture components. The calculated pole densities were plotted in units of time random distribution in which 1 corresponds to complete random orientation distribution. In these graphs, the intersection of the crosshairs corresponds to the extension direction (the ' 3 '-axis), while the ' 2 '-axis represents transverse direction, the ' 1 '-axis the normal direction (see also Figs. 1 and 2, which give the sample direction).

\section{Discussion}

The as-prepared specimen does not show a random orientation of crystallographic planes as one could have expected of a sample that is crystallized from an isotropic melt state. As seen in Fig. 5(a), the texture of the specimen, previous deformation, shows two main components, indicating that upon crystallization from the confined melt between the two metal plates, the molecules acquired some preferred orientation. For clarity, the texture components are labeled I and II in Fig. 6, which is a reproduction of Fig. 5(a). Although there are several distinct grain orientations in each component, they are deemed equivalent for the present study because all the distinct grain orientations belonging to each component are misoriented from the tensile axis in a similar way. Orientation component $I$ is such that the $c$-axis [001], the $a$-axis [100] and the $b$-axis [010] are about 45, 50, and $80^{\circ}$ from the extension axis, respectively. This is relatively the stronger texture component in the initial texture and is comprised of at least three variants (see (100) pole figure in Fig. 5). Orientation component II is such that the $c$ axis, the $a$-axis and the $b$-axis are about 90,75 , and $15^{\circ}$ from the extension axis, respectively. The components I and II are shown schematically in Fig. 8(a) and (b), respectively. Table 1 lists the angles between the extension direction and the crystal axis of components.

The texture in the sample corresponding to the end of the

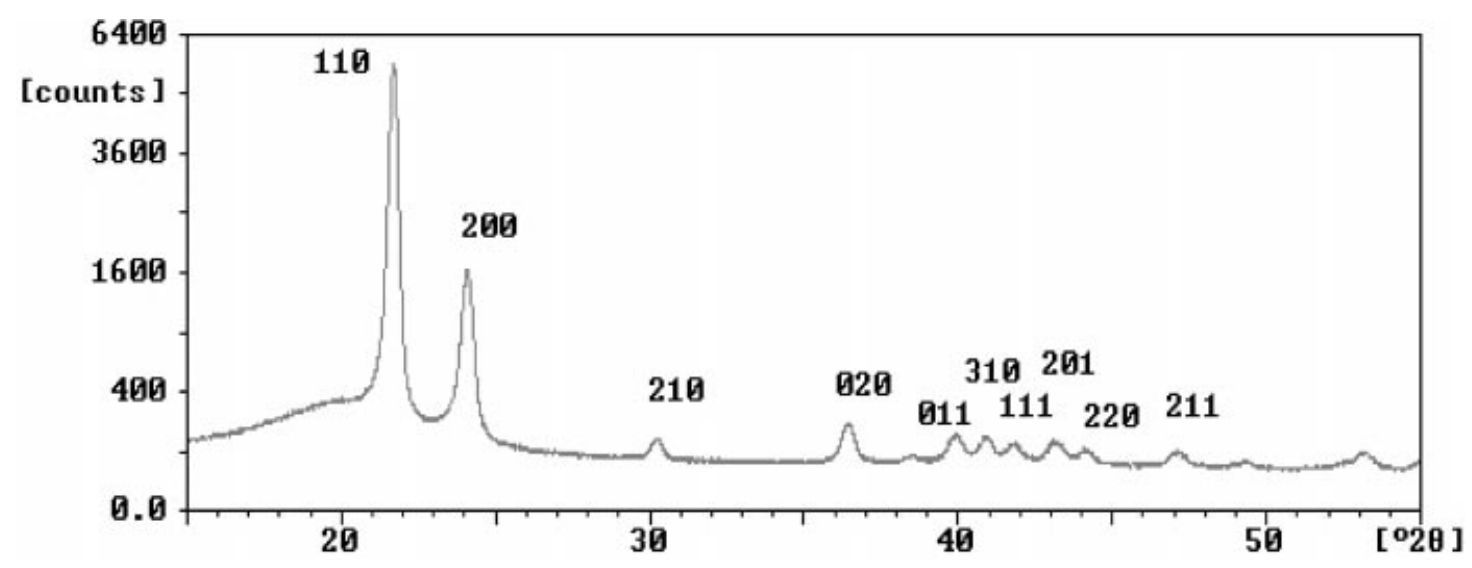

Fig. 4. X-ray diffraction profile of undeformed melt compression HDPE sample. 

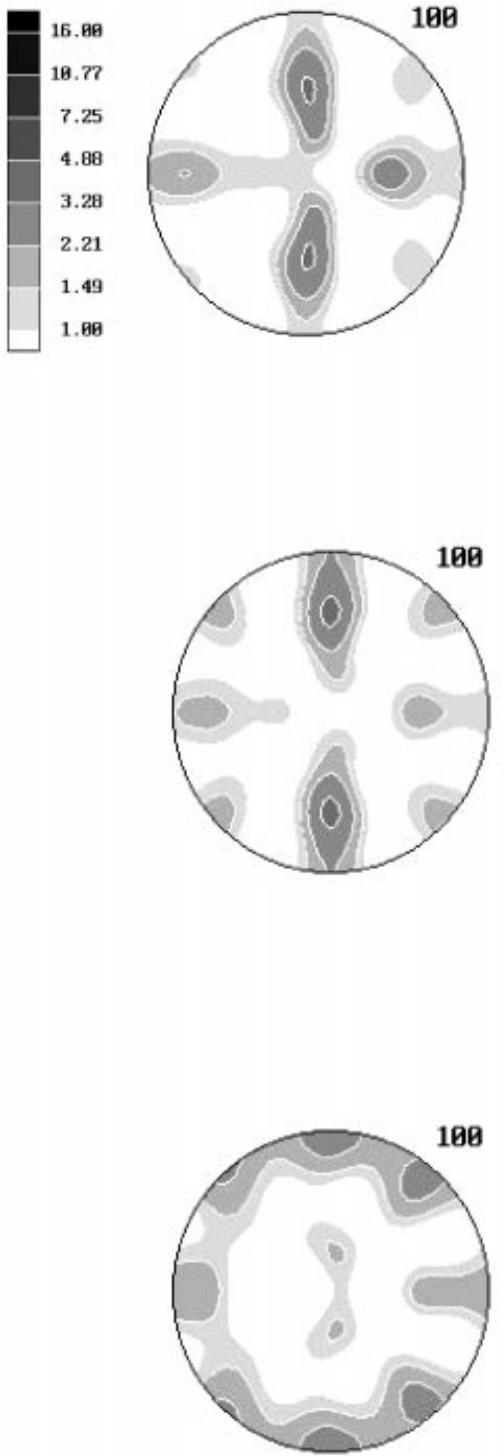

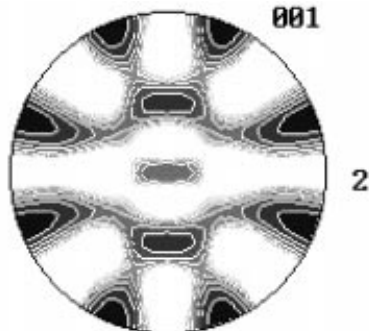

$-1$

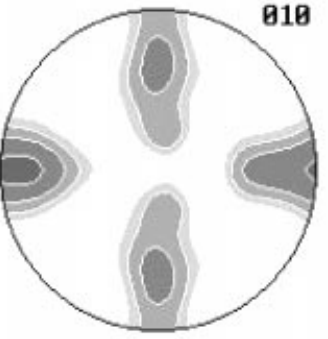

(b)

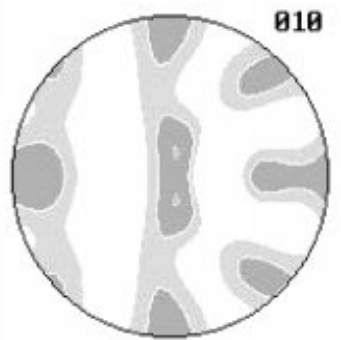

(c)

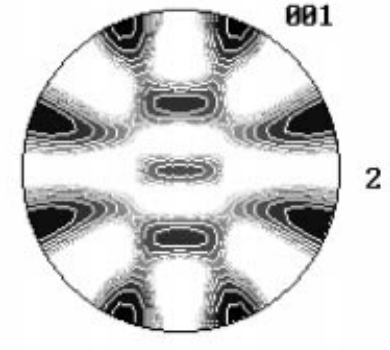

$-1$

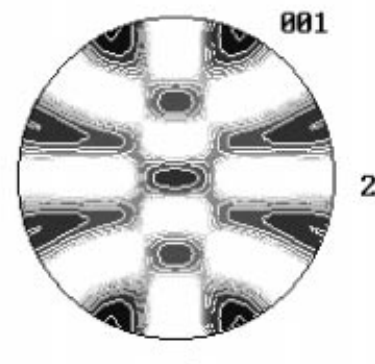

$-1$

Fig. 5. Pole figures of the (100), (010), (001) planes of orthorhombic crystals of linear polyethylene at true strain equals to (a) 0 , (b) 0.11 , (c) 0.7 , (d) 1.2 , (e) 1.8 , (f) 2.1 .

initial elastic regime is shown in Fig. 5(b). Comparison of this pole figure with the initial texture in Fig. 5(a) indicates that there is no major texture change during the elastic regime. Thus, the pole figures agree with the common interpretation that during the elastic region deformation is mainly confined to the amorphous phase [4]. The crystalline lamelli and their orientation remain unchanged.

Fig. 5(c)-(f) document the texture evolution in HDPE to large strains in uniaxial tension. The texture evolution during the regime of plastic flow can be characterized as the development of three additional texture components. They are labeled as components III, IV, and $\mathrm{V}$, respectively, in Fig. 7. This figure is a reproduction of Fig. 5(f) and has been added for clarity and distinction between components. Preferred deformation texture compo- nent III is essentially a $c$-axis (001) fiber component with the $c$-axis aligned with the sample extension direction and the $a$ axis and the $b$-axis lying in the plane perpendicular to the extension direction. The second deformation texture component labeled as IV is a texture component with the (011) axis oriented close to the extension axis (the angle between them is smaller than $5^{\circ}$ ). This component will be hereafter referenced to as a near-(011) component. This near-(011) component can be characterized as a crystal orientation whose $c$-axis, $b$-axis, and $a$-axis are about 25 , 65 , and $90^{\circ}$ to the extension direction, respectively. The component labeled $V$ is a (010) texture component with the $b$-axis aligned with the extension direction. Components III, IV and V are shown schematically in Fig. 8(c)-(e), respectively. 

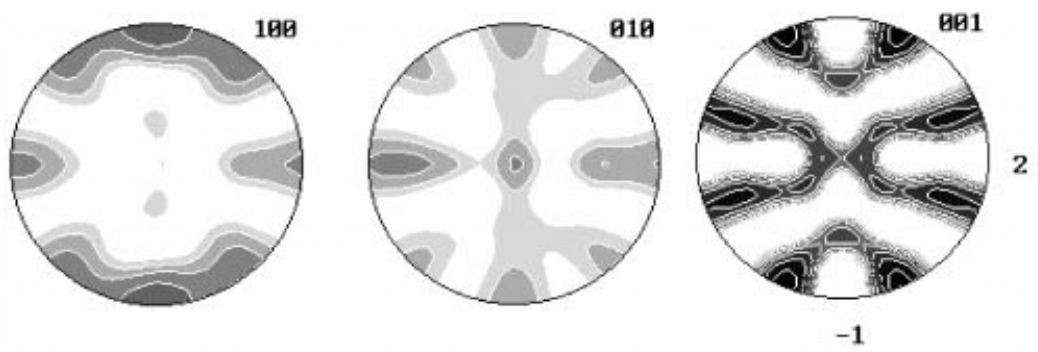

(d)
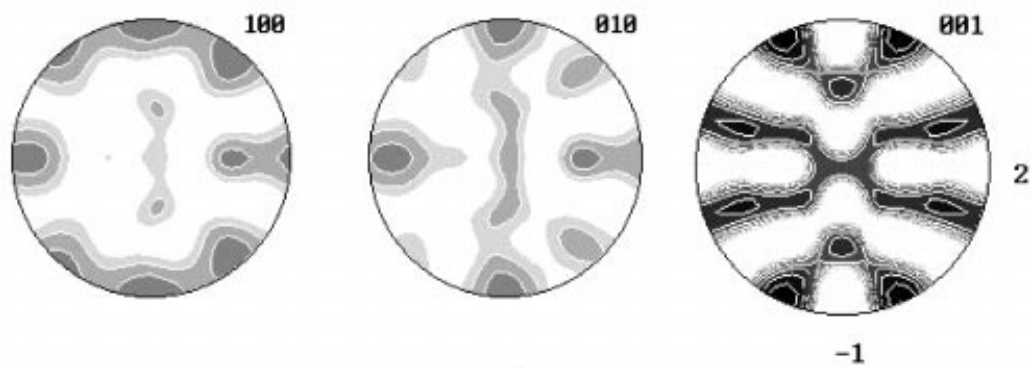

(e)

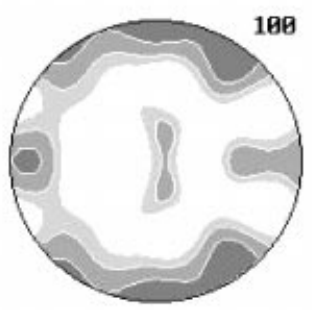

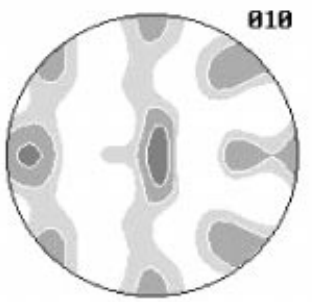

(f)

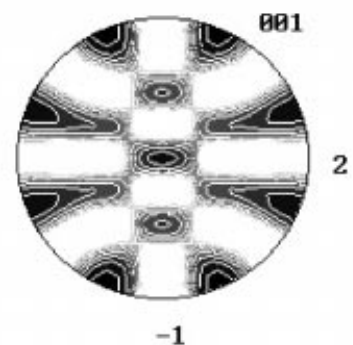

Fig. 5. (continued)

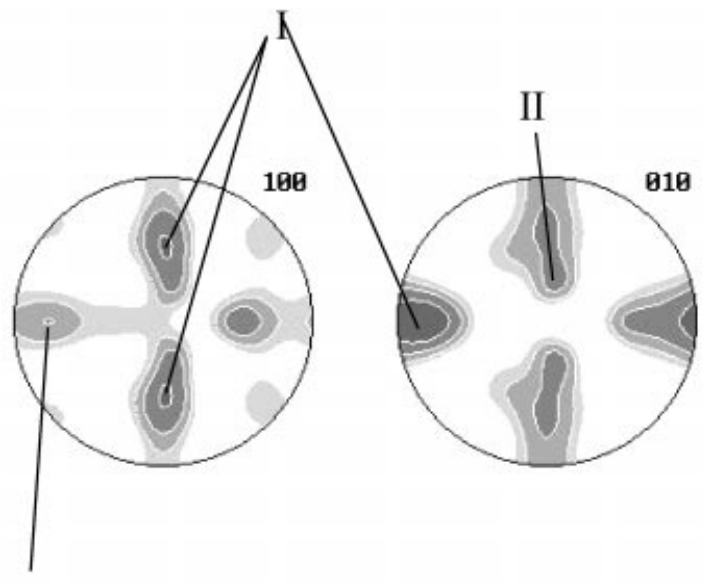

II

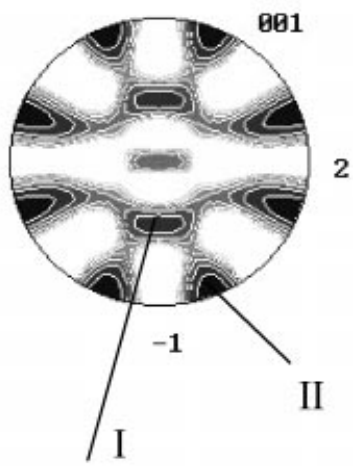

Fig. 6. Same as Fig. 5(a) indicating the two components of texture. 
Table 1

Angles between uniaxial compression axis texture components and unit cell poles

\begin{tabular}{|c|c|c|c|c|c|}
\hline \multirow[t]{2}{*}{ Poles } & \multicolumn{5}{|c|}{ Texture component } \\
\hline & $\mathrm{I}$ & II & III & IV & V \\
\hline$a$-axis & 50 & 75 & 90 & 90 & 90 \\
\hline$b$-axis & 80 & 15 & 90 & 65 & 0 \\
\hline$c$-axis & 45 & 90 & 0 & 25 & 90 \\
\hline
\end{tabular}

There is some similarity between the preferred deformation texture components identified in this study and those identified previously by Krause and Hosford [15]. The difference, however, is that Krause and Hosford report that at about a low strain level of 0.55 , the deformation texture is dominated by the near-(011) component, and by a high strain of 1.8 they report that the deformation texture is essentially dominated by the (001) fiber component. Note that in the present work, at a strain of 0.7 (Fig. 5(c)), both deformation texture components are dominant. Certainly the (001) fiber component in Fig. 5(c) is at least as strong as the (011) component, if not stronger.

Another difference between the measurements reported in this work and those reported previously by Krause and Hosford is that the initial texture components are still prevailing after a true strain of 0.7 . In fact, it is noted that the initial texture components are still present to a significant degree even after a true strain of 2.1. Overall, the measurements indicate a much slower rate of texture evolution compared to the measurements reported by Krause and Hosford. This is particularly puzzling because the initial texture in the sample is not a stable or preferred texture component for the tensile deformation path studied here.
In the undeformed specimen, for component II the $c$-axis is normal to the extension axis and, thus, does not favor the lateral contraction needed to facilitate tensile straining. It should be noted that in metals, where there is a wealth of information on deformation texture evolution, all clues of initial texture are more or less completely wiped out in the material even after strains as low as 0.5 imposed under similar conditions. Therefore, it is quite remarkable that the samples in our study show clear remnants of the initial texture even after very large strain levels.

The differences between the results presented here and those of Krause and Hosford can be attributed to several differences between the two studies: (i) the material, and more importantly the initial texture, in the present study are probably substantially different. Molecular weight or crystallization conditions were not specified in Krause's work. (ii) Rigorous texture analyses techniques have been employed in this study, and (iii) the textures reported here were measured by maintaining the deformation state of the material (i.e. the samples were maintained as constant strain following the mechanical test, while the X-ray measurements were being conducted).

The results presented here pose new challenges to the modeling efforts reported in current literature [10-13,1925]. As described earlier, previously reported models predict the formation of the (001) fiber component (with a slight misalignment in some cases); they do not predict the formation of either the near-(011) component or the weaker (010) component. Furthermore, the models in current literature predict a much faster development of the final texture than what has been observed in the present study. For example, the models of Parks and Ahzi [17] and Lee et al. [13] show a dominant (001) fiber texture component at a true strain of about 1.0. Clearly, this is not the case in the present experiments.

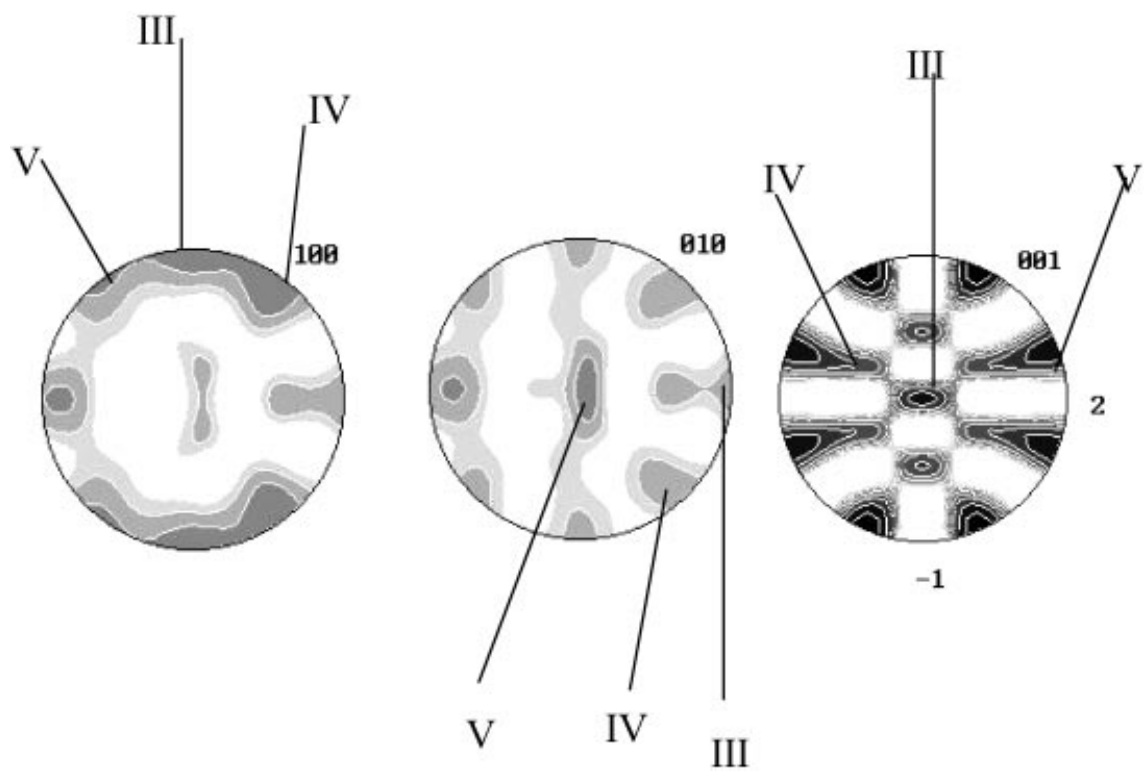

Fig. 7. Same as Fig. 5(f) indicating three additional texture components for samples subject to large strains $(\varepsilon=2.1)$. 


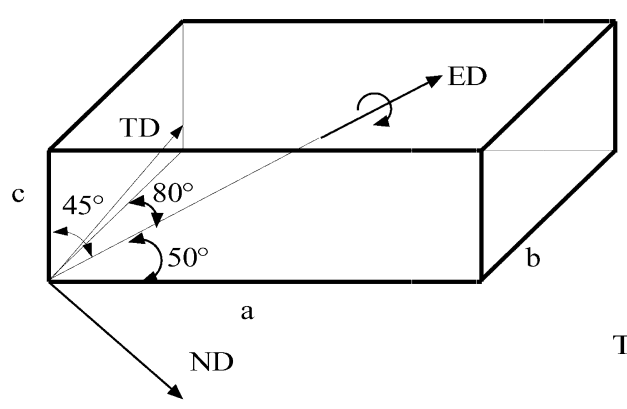

(a)

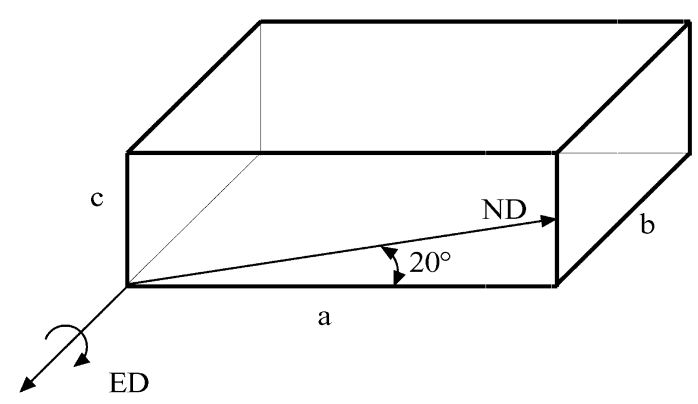

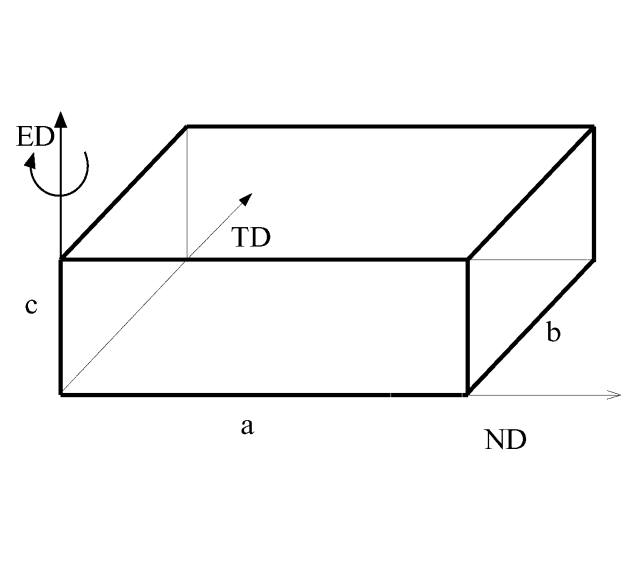

(c)

(e) )

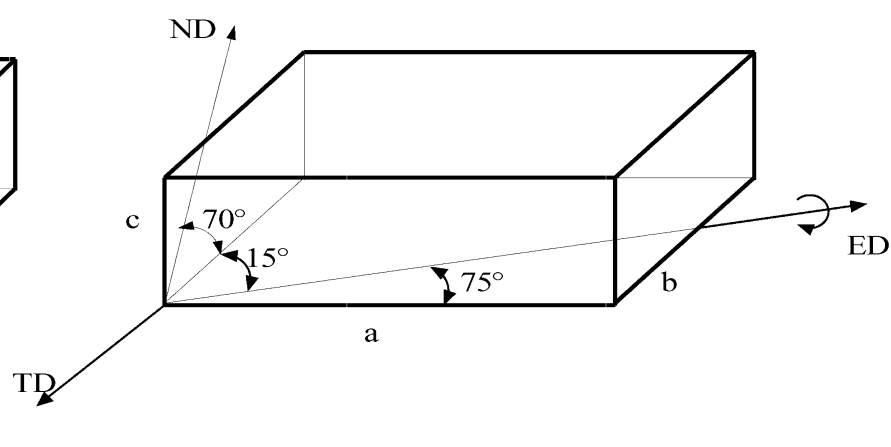

(b)

Fig. 8. Schematic of ideal crystal components (a) component I, (b) component II, (c) component III, (d) component IV, (e) component V. ED, ND, TD represent extension direction, normal direction and transverse direction, respectively, while $a, b, c$ are the crystal axis.

Finally, we address below, possible mechanisms responsible for the observed texture components in rapidly crystallized HDPE under deformation. It should be noted that we have not investigated directly the microscale mechanisms in the present study (this requires highly specialized equipment and detailed investigation). In our opinion, the near(011) component is caused primarily by a combination of chain slip in [001] and transverse slip on [010] directions. Note that in these orientations, the presumed active slip directions are about $45^{\circ}$ with respect to the principal plastic flow direction, i.e. the extension direction. The phenomenology of the active slip directions being oriented at about $35-45^{\circ}$ to the principal plastic flow direction in preferred texture is fairly common in known deformation textures in 

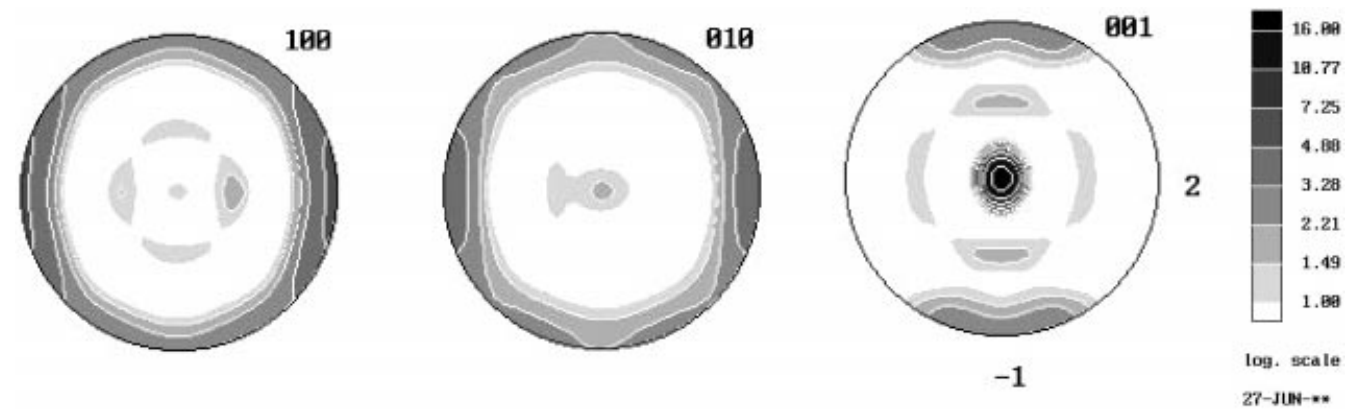

Fig. 9. Pole figure of relaxed sample after strain $=2.1$.

metals (these directions tend to be directions of zero length changes in imposed deformations). For example, the preferred orientation in tensile deformation of fcc metals is a strong (111) fiber component and a weak (100) fiber component. In the (111) fiber component, the active slip directions make an angle of $35^{\circ}$ with respect to the extension axis, while the corresponding angle is $45^{\circ}$ in the (100) fiber component. By analogy, it is reasonable to expect that for extensional deformation of HDPE the chain slip and transverse slip processes produce the (011) component.

It has been argued in other works $[11,15,16]$ that the [001] chain slip produces the (001) component. It is certainly true that a natural consequence of slip on a slip system in tensile loading is to rotate the slip direction towards the tensile direction. However, this does not happen in practice (for example, in the discussion above it was pointed out that the active slip directions are usually at $35-45^{\circ}$ from the extension direction) because the crystals usually experience slip on multiple slip systems and the rotations caused by the activation of the different slip systems cancel out each other when the preferred orientation is achieved. Therefore, in our opinion, it is unlikely that the (001) fiber component is produced by simultaneous chain slip and transverse slip mechanisms. Furthermore, it should also be recognized that once a crystal gets into the (001) orientation, then any combination of chain slip and transverse slip activities cannot produce an extension in the tension direction. Therefore, the (001) fiber orientation is particularly undesirable for the crystal from the viewpoint of producing plastic deformation with minimum energy.

At high deformation levels, it is generally assumed that the spherulitic structure of the crystalline phase breaks down producing crystalline sub-domains that are loosely connected by tie-chains [4]. The physics of this evolution of microstructure are not yet firmly established. However, it is reasonable to assume that these processes have a major influence on texture evolution at large strains. For example, these processes could produce the (001) fiber component that we observe at large strains.

In closing, we address briefly the potential influence of strain relaxation on the texture in deformed samples of HDPE. It is recognized that there are two distinct forms of relaxation in the samples under constant strain (stress relaxation) and under zero stress (strain relaxation). In the present study, although the sample is clamped in the special fixture in the deformed condition, there would be undoubtedly some stress relaxation (i.e. the macroscopic stress in the sample would decrease with time although the overall strain is fixed). Therefore, we first measured the textures in our samples at various time intervals, up to about two weeks, after taking them out from the mechanical testing machines, while keeping them at constant imposed strain levels. The results indicated that there was no significant change in texture in the sample under this sort of relaxation.

In a second set of experiments, we allowed the samples to undergo natural relaxation as soon as the first texture measurement was made as described earlier (in the special fixture shown in Fig. 2). That is, the samples were taken out of the special fixture and allowed to contract and the texture was measured again. The recovery strain was significant in our samples and depended on the amount of initial tensile strain imposed on the sample and the time lag between the mechanical test and when the samples were taken out of the constraining fixture. As expected, the recovery strain was larger for samples subjected to larger tensile strains. Also the recovery strain was lower as more time was allowed between the mechanical test and when the sample was taken out of the constraining fixture. In the sample subjected to a tensile strain of 2.1 , the recovery process reduced the strain by 0.3 to a final strain of 1.8 , just after about three days after the sample was taken out of the fixture. This type of relaxation had an important effect on the texture in the sample (see Fig. 9). The relaxed sample shows a strong $c$-axis fiber texture while all other components described earlier (I, II, IV, and V) have been either eliminated or mitigated substantially. Furthermore, the texture in Fig. 9 corresponds well with that given in previous reports $[14,15]$ suggesting that the analysis in these reports might correspond to "deformed and relaxed" textures and not to "deformed only" textures as reported here. The physics of the texture changes during the relaxation process are not well understood at this time and are well beyond the scope of the present study. These issues are being pursued by our group and will be reported later. 


\section{Conclusions}

The deformation texture evolution in tensile straining of rapidly crystallized HDPE was documented in a careful, systematic, experimental investigation. The textures in the sample at different deformation levels (including the undeformed initial texture) were documented applying rigorous methods of texture analyses, based on spherical harmonics methods, to produce complete, recalculated pole figures. The preferred deformation texture components appear to be (001) (c-axis), near-(011), and (010) components. Although the first one is the strongest deformation texture component, the second component is still growing (although at a relatively slower pace) even at true strains of about 2.1. In general, the rate of texture evolution in the present study was found to be significantly lower than that reported in previous studies. Furthermore, it was found that allowing the sample to relax following the tensile loading has an important influence on the texture in the sample. All of these observations pose new challenges to the modeling efforts in current literature that attempt to predict the concurrent evolution of texture with deformation.

\section{Acknowledgements}

The authors would like to acknowledge the support from the National Science Foundation, Materials Program (DMR 1906-713-32). The authors also acknowledge the support from the Center for Materials Research and Technology (MARTECH) and Center for Nonlinear Non-Equilibrium Phenomena (Cennas). R.G. Alamo acknowledges support from the National Science Foundation, Polymer Program (DMR 94-19508).

\section{References}

[1] Peacock AJ. Handbook of polyethylene: structure, properties and applications. New York, NY: Marcel Dekker, 2000.

[2] Bowden PB, Young RJ. J Mater Sci 1974;9:2034.

[3] Brintzinger HH, Fischer D, Mulhaupt R, Rieger B, Waymouth RM. Angew Chem Int Ed Engl 1995;34:1143.

[4] Lin L, Argon AS. J Mater Sci 1994;29:294.

[5] Termonia I, Smith P. Macromolecules 1987;20:835.

[6] Termonia I, Smith P. Macromolecules 1988;21:2184.

[7] Termonia I, Smith P. Macromolecules 1988;21:3485.

[8] Termonia I, Smith P. Macromolecules 1997;30:5367.

[9] Bunn CW. Trans Faraday Soc 1989;35:483.

[10] Bartczak Z, Galeski A, Argon AS, Cohen RE. Polymer 1996;37:2113.

[11] Bartczak Z, Cohen RE, Argon AS. Macromolecules 1992;25:4692.

[12] Bartczak Z, Argon AS, Cohen RE. Macromolecules 1992;25:5036.

[13] Lee BJ, Argon AS, Parks DM, Ahzi S, Bartczak Z. Polymer 1993;34:3555.

[14] Pazur RJ, Ajji A, Prud'homme RE. Polymer 1993;34:4004.

[15] Krause SJ, Hosford WF. J Polym Sci. Polym Phys Ed 1989;B27:1853.

[16] Butler MF, Donald AM, Ryan AJ. Polymer 1998;39:39.

[17] Taylor GI. J Inst Metals 1938;62:307.

[18] Hughes DJ, Mahendrasingam A, Oatway WB, Heeley EL, Martin C, Fuller W. Polymer 1997;38:6427.

[19] Song HH, Argon AS, Cohen RE. Macromolecules 1990;23:870.

[20] Butler MF, Donald AM, Ryan AJ. Polymer 1998;39:781.

[21] Butler MF, Donald AM. Macromolecules 1998;31:6234.

[22] Young RJ, Bowden PB. Philos Mag 1974;29:1061.

[23] Pazur RJ, Prud'homme RE. Polym.Sci Polym Phys Ed 1994;B32:1475.

[24] Bartczak Z, Argon AS, Cohen RE. Polymer 1994;35:3427.

[25] Butler MF, Donald AM, Bras W, Mant GR. Macromolecules 1995;28:6383.

[26] Hiss R, Hobeika S, Lynn C, Strobl G. Macromolecules 1999;32:4390.

[27] Parks DM, Ahzi S. J Mech Phys Solids 1990;38:701.

[28] Butler JH, Wapp SM, Chambon FH. Adv X-Ray Anal 1999:43.

[29] Quinn Jr FA, Mandelkern L. J Am Chem Soc 1958;80:3178.

[30] Kallend J, Kocks UF, Rollett AD, Wenk HR. Mater Sci Engng A 1991;132:1.

[31] Kocks UF, Tome CN, Wenk HR. Texture and anisotropy. Cambridge: Cambridge University Press, 1998. 